
\title{
3 Research Square \\ Plant Growth-Promoting Endophytic Bacillus Aryabhattai Triggers Novel Genes to Induce Plant Growth
}

\section{Hongli Xu}

Joint R\&D Center of Biotechnology, RETDA, YOTABIO-ENGINEERING CO., LTD.

Jingyao Gao

Joint R\&D Center of Biotechnology, RETDA, YOTABIO-ENGINEERING CO., LTD.

\section{Roxana Portieles}

Joint R\&D Center of Biotechnology, RETDA, YOTABIO-ENGINEERING CO., LTD.

Lihua Du

Joint R\&D Center of Biotechnology, RETDA, YOTABIO-ENGINEERING CO., LTD.

Xiangyou Gao

Joint R\&D Center of Biotechnology, RETDA, YOTABIO-ENGINEERING CO., LTD.

Orlando Borrás-Hidalgo ( $\square$ borrasorlando@yahoo.com )

Joint R\&D Center of Biotechnology, RETDA, YOTABIO-ENGINEERING CO., LTD.

\section{Research Article}

Keywords: Endophytic bacteria, Bacillus aryabhattai, plant growth, transcriptome

Posted Date: December 9th, 2021

DOI: https://doi.org/10.21203/rs.3.rs-1110645/v1

License: (c) (1) This work is licensed under a Creative Commons Attribution 4.0 International License.

Read Full License 


\section{Abstract}

Background: In nature, plants interact with a wide range of microorganisms. Most of these microorganisms have the ability to promote plant growth through the induction of important molecular pathways. The current work evaluated whether the endophytic bacterium Bacillus aryabhattai encourages plant growth and how transcriptional changes might be implicated in this effect.

Results: The endophytic bacterium showed a significant effect on plant growth. Our results revealed that B. aryabhattai promotes the growth of Arabidopsis and tobacco plants. Notably, transcriptional changes in Arabidopsis plants treated with the bacterium were identified. Genes such as cinnamyl alcohol dehydrogenase, apyrase, thioredoxin $\mathrm{H} 8$, benzaldehyde dehydrogenase, indoleacetaldoxime dehydratase, berberine bridge enzyme-like and gibberellin-regulated protein were highly expressed. Additionally, endophytic bacterial genes such as arginine decarboxylase, D-hydantoinase, ATP synthase gamma chain and 2-hydroxyhexa-2,4-dienoate hydratase were activated during the interaction with Arabidopsis.

Conclusions: The results show that new plant growth-related genes are induced during the interaction endophytic bacterium $B$. aryabhattai, and these changes may promote plant growth in sustainable agriculture.

\section{Background}

Plant performance is influenced by the environment and genetic features [1,2]. Under natural conditions, plants are under high pressure from different classes of microorganisms. Interesting relationships that are useful for both partners are established between microorganisms and plants. The cohabitation of microorganisms in the plant has an effect on the growth performance of the plant. These interactions effectively enhance the agricultural properties and yields of plants, as well as the quality of the soil and nutrient cycling $[3,4,5]$.

Additionally, the extensive application of chemical fertilizer has a negative effect on soil quality and the environment [6]. These negative impacts could be reduced with improved farming practices using microbial inoculations such as biofertilizers. Bacterial endophytes are used as biofertilizers to enhance crop production and significantly reduce the impact of chemicals in the environment $[7,8,9]$. Beneficial microorganisms are also used to improve plant yields and constitute sustainable options with respect to chemical fertilizers [10].

Bacillus species comprise the largest class of plant growth-promoting bacteria. Members of the Bacillus genus have the ability to subsist for a long time under adverse environments. Various Bacillus species produce different secondary metabolites capable of inducing plant growth [11]. The use of Bacillus species as biofertilizers provides an alternative for enhancing plant growth and yield [12]. The application of Bacillus species has different effects on plants. Most of these effects are related to the increase in length and biomass of shoots, roots, and leaves $[13,14,15]$. Additionally, Bacillus strains can enhance fruit and grain yields $[16,17]$. 
Endophytic microorganisms produce different bioactive molecules with a marked direct or indirect effect on plant growth. Understanding endophytic microorganism-plant interactions may help clarify the potential to promote plant growth and create a sustainable system for crop production [18]. Previously, $B$. subtilis and $B$. methylotrophicus were involved in the synthesis of plant growth hormones such as indole3-acetic acid, gibberellic acid, 1-aminocyclopropane-1-carboxylate (ACC) deaminase, cytokinins, and spermidines. These proteins are directly involved in the activation of plant growth $[15,19]$. Additionally, $B$. subtilis and $B$. mojavensis can secrete ACC deaminase to inhibit plant senescence $[20,21]$.

Recently, inoculation with Bacillus aryabhattai impressively improved the nutritional status of wheat crops [22]. The growth and productivity of rice were positively enhanced through treatment with $B$. aryabhattai. This bacterium improved the salt tolerance of plants and resulted in more atmospheric nitrogen fixation, phosphate solubilization, and indoleacetic acid production [23]. The $B$. aryabhattai strain also promoted the growth of cowpea via increased indole production, siderophore production, phosphate solubilization, and 1-aminocyclopropane-1-carboxylic acid deaminase activity [24].

B. aryabhattai B8W22 could secrete a large number of organic acids, including oxalic acid, malonic acid, citric acid, succinic acid, indole acetic acid (IAA), and siderophores, that could promote plant growth [25]. Most of the studies involving $B$. aryabhattai were focused on the spectrum of plant growth-promoting secondary metabolites produced by this bacterium. However, additional molecular pathways could be involved in both plants and bacteria during this process.

In general, the understanding of the molecular changes established in plants in response to Bacillus species is still limited. Additionally, investigating the genes expressed in Bacillus during the interaction with plants allows a better understanding of the molecular pathways used by bacteria to induce plant growth. The present study aimed to characterize the effects of the endophytic bacteria $B$. aryabhattai regarding their plant growth-promoting properties and the main molecular pathways involved during the interaction with Arabidopsis plants.

\section{Results}

\section{B. aryabhattai enhances the growth of Arabidopsis and Nicotiana tabacum plants}

Growth promotion in Arabidopsis and $N$. tabacum plants induced by $B$. aryabhattai was tested in the growth room. Arabidopsis plants treated with $B$. aryabhattai showed increased growth compared to the control plants (Fig. 1a). The analysis indicated that bacterial inoculation significantly increased the size of Arabidopsis plants $(4.55 \mathrm{~cm})$ compared with that of control plants $(3.10 \mathrm{~cm})($ Fig. 1b). Regarding the fresh weight of inoculated Arabidopsis plants, the comparison showed that bacterial inoculation significantly enhanced the fresh weight of treated plants $(0.97 \mathrm{~g})$ compared to that of control plants $(0.47$ g) at 20 days posttreatment (Fig. 1C). Moreover, the B. aryabhattai strain induced the growth of $N$. tabacum plants (Fig. 2a). The heights of the inoculated N. tabacum plants were significantly higher (4.05 $\mathrm{cm})$ than those of the uninoculated plants $(2.25 \mathrm{~cm})(\mathrm{Fig} .2 \mathrm{~b})$. Data analysis showed that $N$. tabacum 
plants inoculated with the endophytic bacterium strain produced higher fresh weights $(0.13$ and $0.05 \mathrm{~g}$, respectively) than control plants (Fig. 2c).

\section{B. aryabhattai induces transcriptional changes in genes involved in plant growth}

Endophytic bacterial species frequently have important beneficial effects on plant growth. Genes generally associated with the beneficial effects of endophytic bacteria on plant productivity encode proteins involved in different molecular pathways. To determine whether $B$. aryabhattai induces genes associated with plant growth promotion, RNA-seq was used to evaluate $B$. aryabhattai and Arabidopsis interactions. The range of gene expression changes during the $B$. aryabhattai-Arabidopsis interaction was determined. During the interaction, 21,416 transcripts were identified and annotated (Table S1). From this set, 6,943 new transcripts were annotated (Table S2). The highest number of transcripts (33.05\%) showed an expression level change between 1-and 10-fold. The lowest number of transcripts was found for the highest expression level, >100-fold change (Fig. 3).

Analysis of RNA-seq data during the interaction showed 363 differentially expressed transcripts, which included 268 upregulated and 95 downregulated transcripts (Fig. 4). Among them, cinnamyl alcohol dehydrogenase, apyrase, thioredoxin $\mathrm{H} 8$, benzaldehyde dehydrogenase, indoleacetaldoxime dehydratase, berberine bridge enzyme-like, gibberellin-regulated protein, maturase $\mathrm{K}$, tetratricopeptide repeats (TPR)-like superfamily protein, BTB/POZ, TAZ domain-containing protein and auxin-responsive $\mathrm{GH} 3$ family protein showed the highest differential expression during the $B$. aryabhattai-Arabidopsis interaction. Zinc finger $\mathrm{C}-\mathrm{x} 8-\mathrm{C}-\mathrm{x} 5-\mathrm{C}-\mathrm{x} 3-\mathrm{H}$ type family protein, ankyrin repeat/KH domain protein, CAPRICE-like MYB3, HSP20-like chaperone superfamily protein, gibberellin-regulated protein, abscisic acid 8'-hydroxylase, pectinesterase, agamous-like MADS-box protein, and ethylene-responsive transcription factor were significantly repressed during the interaction (Table 1). 
Table 1

Significant differentially expressed genes during the B. aryabhattai - Arabidopsis interaction ID Log2 Fold Change Description

\section{Upregulated genes}

\begin{tabular}{|c|c|c|}
\hline AT4G37990 & 14983.5 & Cinnamyl alcohol dehydrogenase \\
\hline AT1G14250 & 2685.5 & Apyrase \\
\hline AT1G69880 & 2444.7 & Thioredoxin $\mathrm{H} 8$ \\
\hline AT1G04580 & 2166.9 & Benzaldehyde dehydrogenase \\
\hline AT2G30770 & 2092.8 & Indoleacetaldoxime dehydratase \\
\hline AT1G26390 & 2018.8 & Berberine bridge enzyme-like \\
\hline AT3G02885 & 1666.9 & Gibberellin-regulated protein \\
\hline ATCG00040 & 12.3 & Maturase K \\
\hline ATCG00360 & 12.0 & Tetratricopeptide repeat (TPR)-like superfamily protein \\
\hline AT3G48360 & 10.8 & BTB/POZ and TAZ domain-containing protein \\
\hline AT4G37390 & 10.4 & Auxin-responsive GH3 family protein \\
\hline AT4G37540 & 9.9 & LOB domain-containing protein \\
\hline AT4G28040 & 9.7 & WAT1-related protein \\
\hline AT5G09730 & 9.4 & Beta-D-xylosidase \\
\hline AT1G61120 & 8.6 & $(\mathrm{E}, \mathrm{E})$-geranyllinalool synthase \\
\hline AT1G52400 & 7.8 & Beta-D-glucopyranosyl abscisate beta-glucosidase \\
\hline AT4G21680 & 7.8 & Protein NRT1/ PTR FAMILY \\
\hline AT4G17470 & 7.7 & Alpha/beta-Hydrolases superfamily protein \\
\hline AT1G73220 & 7.5 & Organic cation/carnitine transporter \\
\hline AT3G45140 & 7.3 & Lipoxygenase \\
\hline AT4G15210 & 7.2 & Beta-amylase \\
\hline AT1G54020 & 7.2 & GDSL esterase/lipase \\
\hline ATMG00570 & 7.2 & Sec-independent periplasmic protein translocase \\
\hline AT2G25900 & 6.1 & Zinc finger $\mathrm{CCCH}$ domain-containing protein \\
\hline AT1G21310 & 5.8 & Extensin-3 \\
\hline AT2G23170 & 5.7 & Indole-3-acetic acid-amido synthetase \\
\hline
\end{tabular}




\begin{tabular}{|c|c|c|}
\hline ID & Log2 Fold Change & Description \\
\hline AT3G04070 & 5.6 & NAC transcription factor \\
\hline AT5G67480 & 4.8 & BTB and TAZ domain protein \\
\hline AT1G44350 & 4.7 & IAA-amino acid hydrolase \\
\hline AT1G52000 & 4.4 & Jacalin-related lectin \\
\hline \multicolumn{3}{|c|}{ Downregulated genes } \\
\hline AT1G29560 & 293.1 & Zinc finger $C-x 8-C-x 5-C-x 3-H$ type family protein \\
\hline AT1G12320 & 119.8 & Ankyrin repeat/KH domain protein \\
\hline AT4G01060 & 109.6 & CAPRICE-like MYB3 \\
\hline AT1G76780 & 80.4 & HSP20-like chaperones superfamily protein \\
\hline AT1G74670 & 78.8 & Gibberellin-regulated protein \\
\hline AT2G29090 & 74.5 & Abscisic acid 8'-hydroxylase \\
\hline AT2G47030 & 70.1 & Pectinesterase \\
\hline AT5G60440 & 57.2 & Agamous-like MADS-box protein \\
\hline AT4G16750 & 51.8 & Ethylene-responsive transcription factor \\
\hline AT5G26749 & 48.5 & $\mathrm{C} 2 \mathrm{H} 2$ and $\mathrm{C} 2 \mathrm{HC}$ zinc fingers superfamily protein \\
\hline AT2G40330 & 47.5 & Abscisic acid receptor \\
\hline AT2G36270 & 43.7 & Basic-leucine zipper (bZIP) transcription factor \\
\hline AT5G15800 & 42.6 & MADS-box transcription factor family protein \\
\hline AT5G03680 & 33.4 & Trihelix transcription factor \\
\hline AT1G13920 & 32.9 & Remorin family protein \\
\hline AT3G28830 & 31.3 & Mucin-like protein \\
\hline AT4G31370 & 28.6 & Fasciclin-like arabinogalactan protein \\
\hline AT1G16060 & 23.7 & AP2-like ethylene-responsive transcription factor \\
\hline AT1G01380 & 23.2 & MYB-like transcription factor \\
\hline AT5G57640 & 22.1 & GCK domain-containing protein \\
\hline AT4G33970 & 22.1 & Pollen-specific leucine-rich repeat extensin-like protein \\
\hline AT4G31620 & 18.3 & Transcriptional factor B3 family protein \\
\hline AT5G28640 & 17.8 & GRF1-interacting factor \\
\hline
\end{tabular}




\begin{tabular}{|lll|}
\hline ID & Log2 Fold Change & Description \\
\hline AT3G27650 & 17.2 & LOB domain-containing protein \\
\hline AT4G31380 & 14.0 & Flowering-promoting factor 1-like protein \\
\hline AT5G46530 & 13.4 & AWPM-19-like family protein \\
\hline AT2G45760 & 11.3 & BON1-associated protein \\
\hline
\end{tabular}

Differentially expressed transcripts were analyzed for their function. G0-rich analysis was performed using the annotated differentially expressed genes to generate a list of key genes and the number of genes related to each term. The transcripts with the largest increases in expression were NADH dehydrogenase (quinone) activity, NADH dehydrogenase (ubiquinone) activity, NADH dehydrogenase activity, and oxidoreductase activity (Fig. 5). Based on the KEGG pathway, we categorized the most significant transcripts into indole alkaloid biosynthesis and linoleic acid metabolism pathways (Fig. 6). The expression profile of transcription factor genes shows the transcription-related activities that are enhanced by these genes. To determine their important roles, the transcription factor genes involved in the $B$. aryabhattai-Arabidopsis interaction were analyzed. The largest transcription factor gene families detected in our study were $\mathrm{LOB}$ domain-containing protein, zinc finger $\mathrm{CCCH}$ domain-containing protein, NAC transcription factor, and BTB and TAZ domain protein (Table 1).

During the interaction, a total of 4,305 B. aryabhattai genes were annotated; of these, genes with a log2 fold change between -2.1 and 5.1 were analyzed. Nine and six $B$. aryabhattai genes were up- and downregulated during the interaction with Arabidopsis plants, respectively. The remaining genes had undefined functions, were repeated, had less significant fold changes, or were involved in the primary metabolism of the bacterium. Arginine decarboxylase, D-hydantoinase, membrane protein, ATP synthase gamma chain, and 2-hydroxyhexa-2,4-dienoate hydratase had the highest expression levels in $B$.

aryabhattai during the interaction with Arabidopsis plants. Moreover, putative universal stress protein, Llactate dehydrogenase, succinate dehydrogenase flavoprotein subunit, and glycolate permease were highly downregulated in the bacterium (Table 2) (Table S3). 
Table 2

Major differentially expressed genes in $B$. aryabhattai during the interaction with Arabidopsis plants

\begin{tabular}{|lll|}
\hline ID & Log2 Fold Change * & Swissprot \\
\hline Upregulated genes & \\
\hline gene3107 & 5.1 & Arginine decarboxylase \\
\hline gene1769 & 4.9 & D-hydantoinase \\
\hline gene1522 & 4.9 & Membrane protein \\
\hline gene4310 & 4.8 & ATP synthase gamma chain \\
\hline gene1091 & 4.7 & 2-hydroxyhexa-2,4-dienoate hydratase \\
\hline gene5616 & 3.2 & Transcriptional regulatory protein \\
\hline gene3481 & 2.6 & Microcystinase C \\
\hline gene2400 & 2.4 & 4,4'-diapolycopen-4-al dehydrogenase \\
\hline gene1987 & 2.1 & Succinate-CoA ligase [ADP-forming] subunit beta \\
\hline Downregulated genes & \\
\hline gene4030 & -3.6 & Putative universal stress protein \\
\hline gene5157 & -2.8 & L-lactate dehydrogenase \\
\hline gene1475 & -2.7 & Succinate dehydrogenase flavoprotein subunit \\
\hline gene5156 & -2.6 & Glycolate permease \\
\hline gene4267 & -2.1 & Betaine aldehyde dehydrogenase \\
\hline gene4268 & -2.1 & Alcohol dehydrogenase \\
\hline * Genes with a log2 fold change between -2.1 and 5.1 were included. \\
\hline
\end{tabular}

\section{Discussion}

Several microorganisms display beneficial effects on plants in nature and may be used to enhance growth and crop yields as an alternative to chemical fertilizers. Bacillus species constitute the largest class of growth-promoting bacteria. Bacillus-plant interactions induce plant growth through growthresponsive genes, proteins, phytohormones, and metabolites [11]. Plant growth promotion analyses were performed to evaluate whether $B$. aryabhattai could promote plant growth in different plant species. Arabidopsis (Fig. 1) and N. tabacum (Fig. 2) plants treated with the bacterium showed increased growth, and significant increases were obtained in plant size and fresh weight between the treated and nontreated plants. Notably, although this endophytic bacterium was isolated from wild plant species, it 
exhibited a robust impact on the growth of nonhost plants. The results show that $B$. aryabhattai can enhance the growth of different plant species, thus allowing it to be used to enhance the growth of plants under field conditions.

Diverse bacteria from the Bacillus genus were shown to be good plant growth-promoting bacteria $[11,13$, $19,21,26]$. The first commercial biofertilizer was obtained from Bacillus spp. and enhanced crop yields by $40 \%$ [16]. Additionally, biofertilizers using Bacillus species are more effective at producing diverse metabolite, forming spores, and maintaining cell viability. These characteristics allow the generation of formulated products suited for commercial use [27]. Biofertilizers are good candidates as alternatives to chemical fertilizers to promote plant growth and yields [12]. Additionally, Bacillus species related to roots or rhizospheres could be used to develop biofilms to enhance plant growth [28].

The size and weight of shoots, leaves, and roots from various plant species were enhanced after the application of $B$. insolitus, $B$. subtilis, and $B$. methylotrophicus, respectively $[13,15]$. The production of phytohormones such as indole-3-acetic acid (IAA), cytokinins, gibberellic acid (GA), and spermidines was increased in plants treated with $B$. subtilis and $B$. methylotrophicus and induced plant growth $[15,19,26]$. Additionally, the induction of endogenous proteins, amino acids, and minerals by $B$. megaterium and $B$. methylotrophicus promoted plant growth $[15,29]$. Interestingly, $B$. aryabhattai displayed some plant growth-promoting features, resulting in improved growth of Arabidopsis and $N$. tabacum plants. However, these effects need to be investigated under natural conditions and with crop species. Considering the effect of $B$. aryabhattai on plants, this bacterium could be used to promote plant growth as a biofertilizer under field conditions.

Plant-beneficial bacterial interactions have been extensively analyzed. However, it is not clear which specific molecular pathways are associated with these interactions. This information is important to enhance the potential of these classes of bacteria under field conditions. In the current study, we used RNA sequencing to analyze the genes expressed during Arabidopsis- $B$. aryabhattai interactions. A high number of novel genes involved in metabolite biosynthesis were differentially expressed in our dataset. The results reveal new insights into plant and bacterial gene expression and assist in our understanding of the molecular events implicated during Arabidopsis-B. aryabhattai interactions. Notably, GO and KEGG analyses showed significant changes between treated and nontreated plants. $B$. aryabhattai has a remarkable impact on plants. Our data indicated that $B$. aryabhattai triggered important molecular pathways related to plant growth.

Curiously, cinnamyl alcohol dehydrogenase, apyrase, thioredoxin $\mathrm{H} 8$, benzaldehyde dehydrogenase, indoleacetaldoxime dehydratase, berberine bridge enzyme-like, gibberellin-regulated protein, maturase $\mathrm{K}$, tetratricopeptide repeat (TPR)-like superfamily protein, BTB/POZ and TAZ domain-containing protein and auxin-responsive GH3 family protein genes were highly induced during the Arabidopsis- $B$. aryabhattai interaction.

Cinnamyl alcohol dehydrogenase is a key enzyme during plant secondary metabolism, especially lignin synthesis, and it is closely related to plant growth and development. Lignin constitutes one of the major 
components of plant cell walls and has the function of connecting cells. Previously, this enzyme was expressed in lateral roots and in root tips in sweet potato, and its activity was induced by abscisic acid [30]. Additionally, cinnamyl alcohol dehydrogenase genes are related to lignin biosynthesis during the final developmental phases of soybean seeds [31]. Most likely, B. aryabhattai promotes the synthesis of lignin during the growing phase of Arabidopsis and $N$. tabacum plants, resulting in the robust phenotype observed for the plants treated with the bacterium.

Likewise, the extracellular nucleotides can be regulated by apyrases. Apyrases are involved in the control of plant growth and development. Specifically, apyrases have influence in the auxin transport and stomatal aperture. Removal of the apyrases activity can leads to growth inhibition [32]. Potato plants with apyrase gene silenced showed phenotypic changes, retardation of growth, increasing of tuber number per plant, and effect on tuber morphology [33]. Meanwhile, the expression of apyrases gene in Arabidopsis plants had a marked effect on the growth of plant tissues and accumulation of auxin levels $[34,35]$. Thus, there was significant evidence that apyrases developed a crucial role in regulating the growth of Arabidopsis and N. tabacum plants.

Besides, benzaldehyde dehydrogenase is an important enzyme involves in the processing of benzaldehyde to benzoic acid. The growth, mineral composition, and chlorophyll content of soybean plants were influenced by benzoic acid [36]. Benzoic acid had a remarked effect on the growth and yield of tomato plants. Additionally, there was a positive effect of benzoic on fruit yield [37]. Interestingly, $B$. aryabhattai could be indirectly inducing the plant growth through this enzyme, which displays a key role in the benzoic acid pathway.

Gibberellins are involved in plant growth, development processes, stem elongation, flowering, and seed germination [38]. Vegetative and reproductive growth were severely affected in rice plants expressing a gibberellin-regulated gene in antisense orientation [39]. In addition, maturase $\mathrm{K}$ gene was highly expressed in Anoectochilus roxburghii plants treated with endophytic fungi [40]. Recently, the maturase $\mathrm{K}$ gene was induced during the Arabidopsis - Bacillus altitudinis interaction [41].

Further, proteins with tetratricopeptide repeat motifs are basic components for gibberellin and ethylene responses. A silencing of an Arabidopsis chloroplast-localized tetratricopeptide repeat protein gene affected the plant growth, leaf greening, chloroplast, and genes involved in photosynthesis [42]. Recently, an endophytic $B$. altitudinis induced Arabidopsis tetratricopeptide repeat-like superfamily proteins genes with a marked effect on plant growth [41]. Moreover, broad-complex, tram track, and bric-a-brac family proteins (BTB) genes had a high influence on transcription, protein modification, chromatin, cytoskeletal, and hormone pathways in tomato [43]. These kind of genes could be implicated indirectly in the activation of phytohormone related with plant growth.

The bacterium $B$. aryabhattai induced the plant growth by the trigger of key molecular pathways, involved in the production of phytohormones and transcription factors. The root development, shoot growth, and fruit ripening were regulated by Aux/IAA family genes [44]. Auxin influences numerous stages of plant 
development and growth by directing the expression of auxin-activate genes [45]. Auxin controls plant development and growth by changing the induction of different genes [46].

While much of what happens in the plant during interactions with endophytic bacteria is known, it is also important to understand what happens in the bacteria during its interaction with the plant, such as which genes are expressed in the bacterium that may contribute to the growth phenotype of treated plants. Interestingly, arginine decarboxylase, D-hydantoinase, ATP synthase gamma chain and 2-hydroxyhexa2,4-dienoate hydratase genes were highly induced in $B$. aryabhattai during the interaction with the plant, which constitutes the first evidence of the expression of these kinds of genes in this species. We speculate that the overexpression of these genes in B. aryabhattai might enhance plant growth.

The activity of arginine decarboxylase was implicated in the effect of hormones on plant growth [47]. Arginine decarboxylase is an important enzyme responsible for putrescine biosynthesis. Arginine decarboxylase expression correlates with cell growth and stress responses in apple plants [48]. This enzyme is involved in efficient ROS elimination and its influence on root growth, which is conducive to drought tolerance [49].

In addition, an active D-hydantoinase from Pseudomonas fluorescens was related to the synthesis of Damino acids [50]. Although plants are constantly exposed to D-amino acids (D-AAs) in the rhizosphere, these compounds have inhibitory effects on plant growth. A recent characterization of D-AA-stimulated ethylene production in Arabidopsis showed the physiological function of a specific D-AA and its metabolizing enzyme in plants [51]. Most likely, the regulation of plant D-AA content could influence the composition of the rhizosphere [52].

\section{Conclusions}

Our results showed that the endophytic bacterium B. aryabhattai induced the growth of Arabidopsis and $N$. tabacum plants. Notably, new genes involved in different plant growth pathways were identified during the Arabidopsis- $B$. aryabhattai interaction. The expression of these genes could be directly or indirectly related to the phenotype of the plants treated with the bacterium. On the other hand, genes expressed in the bacterium during the interaction with the plant could also have an influence on the molecular mechanisms activated in the plant. However, the effect of this bacterium under natural conditions and on important crops needs to be evaluated. The growth phase of plants is regulated by the expression of many genes with different roles. The analysis of their function is important but complex, as each may have a specific, unique role. For this reason, the evaluation of gene function in the bacterium could provide an important approximation of the involvement of these genes during plant interactions and their relationship with the activation of growth-promoting genes in the plant. This line of inquiry should be investigated further. Finally, the potential beneficial effects of $B$. aryabhattai found here suggest that this bacterium is an appropriate and efficient candidate for use in sustainable agriculture.

\section{Methods}




\section{Endophytic bacterium, plant materials, and growth conditions}

The Bacillus aryabhattai bacterium was previously isolated and identified from the wild plant species $G$. chinensis (Keng) according to Portieles et al. [53]. Samples were collected from the wild plant species $G$. chinensis (Keng) along the Fu Tuan River ( $35^{\circ} 20^{\prime} 17^{\prime \prime} \mathrm{N}, 119^{\circ} 26^{\prime}$ ' $^{\prime \prime} \mathrm{E}$ ) within $5 \mathrm{~km}$ of the coastal region of Rizhao city in Shandong Province, People's Republic of China. G. chinensis (Keng) was identified according to the data on morphological traits from the Flora of China (http://www.iplant.cn/foc/). This experimental study complies with Chinese national and local laws, and sample collection was permitted by the Rizhao Administration and Municipal Sciences and Technology Department. (Collection information: South China Botanical Garden (IBSC) of the Chinese Academy of Sciences. Source: China Digital Plant Specimens Museum. Identifier: 0114164. Collector: Zhang Zhisong Acquisition number: 401467). The strain was cultivated in Luria-Bertani (LB) agar medium (yeast extract, $5 \mathrm{~g} / \mathrm{l}$ : peptone, 10 $\mathrm{g} / \mathrm{l}$; sodium chloride, $5 \mathrm{~g} / \mathrm{l}$; agar, $12 \mathrm{~g} / \mathrm{l} ; \mathrm{pH} 7$ ) at $37^{\circ} \mathrm{C}$. Seeds of the Arabidopsis thaliana ecotype Columbia were surface-sterilized and placed on Murashige and Skoog (0.5X MS) basal media (Sigma Aldrich, St Louis, MO, USA) supplemented with $1 \% \mathrm{w} / \mathrm{v}$ sucrose. The seeds were kept at $4^{\circ} \mathrm{C}$ in the dark for 2 days and transferred to a growth room under a $16 \mathrm{~h}$ light/ $8 \mathrm{~h}$ dark photoperiod at $22^{\circ} \mathrm{C}$. Small plants were sown in a substrate composed of peat plugs and vermiculite (1:1) for 14 days. In addition, Nicotiana tabacum seeds were germinated, and the plants were grown in 6-inch pots containing sterilized black turf and rice husk (4:1) substrate and maintained in a growth room at $23^{\circ} \mathrm{C}$. All the substrates used were sterilized at $120^{\circ} \mathrm{C}$ for 20 minutes.

\section{Greenhouse experiments}

Bacillus aryabhattai was cultivated in $100 \mathrm{~mL}$ of LB broth medium in a 250-ml Erlenmeyer flask with shaking (200 rpm) for one day at $37^{\circ} \mathrm{C}$ in the dark. The optical density (OD) of the B. aryabhattai fermentation product was adjusted to $1.0\left(4.77 \times 10^{9} \mathrm{CFU} / \mathrm{mL}\right)$, and $30 \mathrm{~mL}$ of the fermentation product was applied per pot. Three plants were grown per plastic pot in a growth room at $25^{\circ} \mathrm{C}$ and irrigated with water without any fertilizers. Arabidopsis thaliana and Nicotiana tabacum (five days old) plants were treated with the $B$. aryabhattai fermentation product twice weekly for one month. The plant size and fresh weight parameters were evaluated after one month of treatment with $B$. aryabhattai. A completely randomized pot experiment with five replicates for each treatment was performed to analyze the influence of $B$. aryabhattai on the growth of Arabidopsis thaliana and Nicotiana tabacum plants. Data were analyzed using GraphPad Prism software (La Jolla, CA, USA). Significant differences among the mean values were determined using a t test at $P<0.05$. Five replicates were used for each treatment. The experiments were replicated three times.

\section{Identification Of New Differentially Expressed Genes Using Rna Sequencing}


Five-day-old Arabidopsis plants were treated with $30 \mathrm{~mL}\left(4.77 \times 10^{9} \mathrm{CFU} / \mathrm{mL}\right)$ of $B$. aryabhattai. The bacterium was well established $72 \mathrm{~h}$ post-inoculation. Leaves, stems, and roots from five plants were collected after establishment. Plants treated with water were used as a control. Treatments and controls were repeated three times per group. Total RNA was isolated using the Qiagen RNeasy Midi Kit (Hilden, Germany), and the concentration of total RNA was determined using spectrometry. After isolation of the total RNA, eukaryotic mRNA was enriched using oligo (dT) beads. The samples were sequenced using an Illumina HiSeq ${ }^{\text {TM }} 2000$ (Personalbio Co. (Shanghai, People's Republic of China). High-quality reads were processed using the Perl script, and the differentially expressed genes were identified using the edgeR package [54]. Genes with a fold change $\geq 2$ were considered significantly differentially expressed genes. Gene Ontology (GO) and Kyoto Encyclopedia of Genes and Genomes (KEGG) pathway enrichment analyses were used to characterize the differentially expressed genes $[55,56]$. In addition, the GO annotations were analyzed using Blast2GO software [57, 58].

The prokaryote genetic analysis process involved filtering the raw data to obtain a high-quality sequence and comparing the filtered sequences to the reference genome for the species [59, 60]. HTSeq was used to compare the read count values from each gene to the original expression level of that gene [61]. To make gene expression levels comparable between different genes and samples, we normalized the sequencing depth and gene length using fragments per kilobase of transcript per million.

\section{Declarations}

\section{Authors' contributions}

Orlando Borrás-Hidalgo, Hongli Xu and Xiangyou Gao conceived the study. Orlando Borrás-Hidalgo and Hongli Xu designed the experiments. Hongli Xu, Jingyao Gao,Roxana Portieles, Lihua Du, Xiangyou Gao, and Orlando Borrás-Hidalgo performed the experiments. Hongli Xu and Orlando Borrás-Hidalgo assisted with the data analysis. Orlando Borrás-Hidalgo wrote the article.

\section{Authors' information}

Not applicable

\section{Funding}

This study was supported by the Special Funds for Guiding Local Science and Technology Development of Central Government of Shandong Province (No. YDZX20193700004362).

\section{Availability of data and materials}

The BioProject accession numbers: BioProject ID PRJNA781678 and BioProject ID PRJNA781727.

\section{Declarations}

\section{Ethics approval and consent to participate}


No live fish were handled during this study.

\section{Consent for publication}

Not applicable

\section{Competing interests}

The authors declare that the research was conducted in the absence of any commercial or financial relationships that could be construed as a potential conflict of interest.

\section{Author details}

1 Joint R\&D Center of Biotechnology, RETDA, YOTABIO-ENGINEERING CO., LTD., 99 Shenzhen Road, Rizhao, 276826, Shandong, P.R. China. ${ }^{2}$ State Key Laboratory of Biobased Material and Green Papermaking, Shandong Provincial Key Lab of Microbial Engineering, Qilu University of Technology (Shandong Academy of Science), Jinan, P.R. China

\section{References}

1. Kleinwechter, U., Gastelo, M., Ritchie, J., Nelson, G. \& Asseng, S. Simulating cultivar variations in potato yields for contrasting environments. Agric. Syst, 145, 51-63 (2016).

2. Li, C. et al. Numerous genetic loci identified for drought tolerance in the maize nested association mapping populations. BMC Genomic, 17, 894 (2016).

3. Soliman, M. et al. Exogenous nitric oxide mitigates nickel-induced oxidative damage in Eggplant by upregulating antioxidants, osmolyte metabolism, and glyoxalase systems. Plants, 8, 562 (2019).

4. Habib, N. et al. Use of nitric oxide and hydrogen peroxide for better yield of wheat (Triticum aestivum L.) under water deficit conditions: growth, osmoregulation, and antioxidative defense mechanism. Plants, 9, 285 (2020).

5. Elkelish, A. et al. Exogenous ascorbic acid induced chilling tolerance in tomato plants through modulating metabolism, osmolytes, antioxidants, and transcriptional regulation of catalase and heat shock proteins. Plants, 9, 431 (2020).

6. Azab, E., Elsalam, H. \& Sharnouby, M. Performance of Catharanthus Roseus Plants in Response to Gamma Irradiation. J. Biol. Chem. Res, 33, 130-140 (2016).

7. Afzal, I., Shinwari, Z. K., Sikandar, S. \& Shahzad, S. Plant beneficial endophytic bacteria: Mechanisms, diversity, host range and genetic determinants. Microbiol. Res, 221, 36-49 (2019).

8. Alkahtani, M. D. et al. Isolation and characterization of plant growth promoting endophytic bacteria from desert plants and their application as bio-inoculants for sustainable agriculture. Agronomy, 10, 1325 (2020).

9. Hassan, S. E. D. Plant growth-promoting endophytic bacterial community inhabiting the leaves of Pulicaria incisa (Lam.) DC inherent to arid regions. Plants, 10, 76 (2021). 
10. Ke, J., Wang, B. \& Yoshikuni, Y. Microbiome engineering: synthetic biology of plant-associated microbiomes in sustainable agriculture. Trends Biotechnol, 39, 244-261 (2021).

11. Radhakrishnan, R., Hashem, A. \& Abd Allah, E. F. Bacillus: a biological tool for crop improvement through bio-molecular changes in adverse environments.Front. Physiol. 2017;8 667

12. Choudhary, D. K. Plant growth promotion (PGP) activities and molecular characterization of rhizobacterial strains isolated from soybean (Glycine max L. Merril) plants against charcoal rot pathogen, Macrophomina phaseolina. Biotechnol. Lett, 33, 2287-2295 (2011).

13. Ashraf, M., Hasnain, S., Berge, O. \& Mahmood, T. Inoculating wheat seedlings with exopolysaccharide-producing bacteria restricts sodium uptake and stimulates plant growth under salt stress. Biol. Fertil. Soils, 40, 157-162 (2004).

14. Barnawal, D., Maji, D., Bharti, N., Chanotiya, C. S. \& Kalra, A. ACC deaminase-containing Bacillus subtilis reduces stress ethylene-induced damage and improves mycorrhizal colonization and rhizobial nodulation in Trigonella foenumgraecum under drought stress. J. Plant Growth Regul, 32, 809-822 (2013).

15. Radhakrishnan, R. \& Lee, I. J. Gibberellins producing Bacillus methylotrophicus KE2 supports plant growth and enhances nutritional metabolites and food values of lettuce. Plant Physiol. Biochem, 109, 181-189 (2016).

16. Kilian, M. et al. FZB24R Bacillus subtilis - mode of action of a microbial agent enhancing plant vitality. Pflanzenschutz Nachr. Bayer, 1, 72-93 (2000).

17. Dursun, A., Ekinci, M. \& Donmez, M. F. Effects of foliar application of plant growth promoting bacterium on chemical contents, yield and growth of tomato (Lycopersicon esculentum L.) and cucumber (Cucumis sativus L.). Pak. J. Bot, 42, 3349-3356 (2010).

18. Hassan, S. E. D. Plant growth-promoting activities for bacterial and fungal endophytes isolated from medicinal plant of Teucrium polium L. J. Adv. Res, 8, 687-695 (2017).

19. Xie, S. et al. Plant growth promotion by spermidine-producing Bacillus subtilis OKB105. Mol. Plant Microbe Interact, 27, 6556663 (2014).

20. Xu, M. et al. Bacterial community compositions of tomato (Lycopersicum esculentum Mill.) seeds and plant growth promoting activity of ACC deaminase producing Bacillus subtilis (HYT-12-1) on tomato seedlings. World J. Microbiol. Biotechnol, 30, 835-845 (2014).

21. Pourbabaee, A. A., Bahmani, E., Alikhani, H. A. \& Emami, S. Promotion of wheat growth under salt stress by halotolerant bacteria containing ACC deaminase. J. Agric. Sci. Technol, 18, 855-864 (2016).

22. Hussain, A. et al. Plant-growth-promoting Bacillus and Paenibacillus species improve the nutritional status of Triticum aestivum L. PLoS One, 15 (12), e0241130 (2020).

23. Sultana, S. et al. Isolation and identification of salt-tolerant plant-growth-promoting rhizobacteria and their application for rice cultivation under salt stress. Can. J. Microbiol, 66 (2), 144-160 (2020).

24. Inthama, P., Pumas, P., Pekkoh, J., Pathom-Aree, W. \& Pumas, C. Plant growth and drought tolerancepromoting bacterium for bioremediation of Paraquat pesticide residues in agriculture soils. Front. 
Microbiol, 12, 604662 (2021).

25. Song, S., Liu, Y., Wang, N. R. \& Haney, C. H. Mechanisms in plant-microbiome interactions: lessons from model systems. Curr. Opin. Plant Biol, 2, 102003 (2021).

26. Fouda, A. et al. Plant growth-promoting endophytic bacterial community inhabiting the leaves of Pulicaria incisa (Lam.) DC inherent to arid regions. Plants, 10, 76 (2021).

27. Haas, D. \& Defago, G. Biological control of soil-borne pathogens by fluorescent pseudomonads. Nat. Rev. Microbiol, 3, 307-319 (2005).

28. Beauregard, P. B., Chai, Y., Vlamakis, H., Losick, R. \& Kolter, R. Bacillus subtilis biofilm induction by plant polysaccharides. Proc. Natl. Acad. Sci. U.S.A. 2013; 110: 1621-E1630

29. Kang, S. M. et al. Phosphate solubilizing Bacillus megaterium mj1212 regulates endogenous plant carbohydrates and amino acids contents to promote mustard plant growth. Indian J. Microbiol, 54, 427-433 (2014).

30. Kim, Y. H., Bae, J. M. \& Huh, G. H. Transcriptional regulation of the cinnamyl alcohol dehydrogenase gene from sweet potato in response to plant developmental stage and environmental stress. Plant Cell Rep, 29 (7), 779-91 (2010).

31. Baldoni, A., Von Pinho, E. V., Fernández, J. S., Abreu, V. M. \& Carvalho, M. L. Gene expression in the lignin biosynthesis pathway during soybean seed development. Genet Mol Res, 12 (3), 2618-24 (2013).

32. Clark, G. B., Morgan, R. O., Fernandez, M. P., Salmi, M. L. \& Roux, S. J. Breakthroughs spotlighting roles for extracellular nucleotides and apyrases in stress responses and growth and development. Plant Sci, 225, 107-16 (2014).

33. Riewe, D., Grosman, L., Fernie, A. R., Wucke, C. \& Geigenberger, P. The potato-specific apyrase is apoplastically localized and has influence on gene expression, growth, and development. Plant Physiol, 147 (3), 1092-109 (2008).

34. Wu, J. et al. Apyrases (nucleoside triphosphate-diphosphohydrolases) play a key role in growth control in Arabidopsis. Plant Physiol, 144 (2), 961-75 (2007).

35. Liu, X. et al. Role for apyrases in polar auxin transport in Arabidopsis. Plant Physiol, 160 (4), 198595 (2012).

36. Baziramakenga, R., Simard, R. R. \& Leroux, G. D. Effects of benzoic and cinnamic acids on growth, mineral composition, and chlorophyll content of soybean. J. Chem. Ecol, 20, 2821-2833 (1994).

37. Benavides-Mendoza, A., Burgos-Limón, D., Ramirez, H., Robledo-Torres, V. \& Sandoval-Rangel, A. Benzoic acid effect in the growth and yield of tomato in calcareous soil. Acta Hort, 938 (1), 251-256 (2012).

38. Jan, A. \& Komatsu, S. Functional characterization of gibberellin-regulated genes in rice using microarray system. Genomics Proteomics Bioinformatics, 4 (3), 137-44 (2006).

39. Jan, A., Kitano, H., Matsumoto, H. \& Komatsu, S. The rice OsGAE1 is a novel gibberellin-regulated gene and involved in rice growth. Plant Mol Biol, 62 (3), 439-52 (2006). 
40. Ye, B. et al. Beneficial effects of endophytic fungi from the Anoectochilus and Ludisia Species on the growth and secondary metabolism of Anoectochilus roxburghii. ACS Omega, 5 (7), 3487-3497 (2020).

41. Zhang, D. et al. Borrás-Hidalgo O. Endophytic Bacillus altitudinis strain uses different novelty molecular pathways to enhance plant growth. Front. Microbiol, 12, 692313 (2021).

42. Ma, F. et al. A novel tetratricopeptide repeat protein, WHITE TO GREEN1, is required for early chloroplast development and affects RNA editing in chloroplasts. J. Exp. Bot, 68, 5829-5843 (2017).

43. Li, J. et al. Genome-wide identification and expression analysis of the BTB domain-containing protein gene family in tomato. Genes Genomics, 40 (1), 1-15 (2018).

44. Luo, J., Zhou, J. J. \& Zhang, J. Z. Aux/IAA gene family in plants: molecular structure, regulation, and function. Int. J. Mol. Sci, 19 (1), 259 (2018).

45. Jiang, W. et al. Genome-wide identification, characterization analysis and expression profiling of auxin-responsive GH3 family genes in wheat (Triticum aestivum L.). Mol. Biol. Rep, 47 (5), 38853907 (2020).

46. Jain, M., Kaur, N., Tyagi, A. K. \& Khurana, J. P. The auxin-responsive GH3 gene family in rice (Oryza sativa). Funct. Integr. Genomics, 6 (1), 36-46 (2006).

47. Apelbaum, A., Goldlust, A. \& Icekson, I. Control by ethylene of arginine decarboxylase activity in pea seedlings and its implication for hormonal regulation of plant growth. Plant Physiol, 79 (3), 635-40 (1985).

48. Hao, Y. J., Kitashiba, H., Honda, C., Nada, K. \& Moriguchi, T. Expression of arginine decarboxylase and ornithine decarboxylase genes in apple cells and stressed shoots. J. Exp. Bot, 56 (414), 1105-15 (2005).

49. Wang, J. et al. An arginine decarboxylase gene PtADC from Poncirus trifoliata confers abiotic stress tolerance and promotes primary root growth in Arabidopsis. J. Exp. Bot, 62 (8), 2899-914 (2011).

50. Xu, G. C., Li, L., Han, R. Z., Dong, J. J. \& Ni, Y. Characterization and soluble expression of Dhydantoinase from Pseudomonas fluorescens for the synthesis of D-amino acids. Appl. Biochem. Biotechnol, 179 (1), 1-15 (2016).

51. Kolukisaoglu, Ã. D-amino acids in plants: sources, metabolism, and functions. Int. J. Mol. Sci, 21 (15), 5421 (2020).

52. Hener, C., Hummel, S., Suarez, J., Stahl, M. \& Kolukisaoglu, Ã. d-Amino acids are exuded by Arabidopsis thaliana roots to the rhizosphere. Int. J. Mol. Sci, 19 (4), 1109 (2018).

53. Portieles, R. et al. Heat-killed endophytic bacterium induces robust plant defense responses against important pathogens. Sci. Rep, 11 (1), 12182 (2021).

54. Robinson, M. D., McCarthy, D. J. \& Smyth, G. K. edgeR: a Bioconductor package for differential expression analysis of digital gene expression data. Bioinformatics, 26 (1), 139-140 (2010).

55. Ashburner, M. et al. Gene Ontology: tool for the unification of biology. Nat. Genet, 25, 25-29 (2000). 
56. Kanehisa, M. \& Goto, S. KEGG: Kyoto Encyclopedia of Genes and Genomes. Nucleic Acids Res, 28, 27-30 (2000).

57. Altschul, S. F. et al. Gapped BLAST and PSIBLAST: A new generation of protein database search programs. Nucleic Acids Res, 25, 3389-3402 (1997).

58. Conesa, A. et al. Blast2GO: A universal tool for annotation, visualization and analysis in functional genomics research., 2, 3674-3676 (2005).

59. Delcher, A. L., Harmon, D., Kasif, S., White, O. \& Salzberg, S. L. Improved microbial gene identification with GLIMMER. Nucleic acids research, 27 (23), 4636-4641 (1999).

60. Coil, D., Jospin, G. \& Darling, A. E. A5-MiSeq: an updated pipeline to assemble microbial genomes from Illumina MiSeq data. Bioinformatics, 31 (4), 587-589 (2014).

61. Simon, A., Theodor, P. \& Huber, W. HTSeq-a Python framework to work with high-throughput sequencing data., 31, 166-169 (2015).

\section{Figures}


A

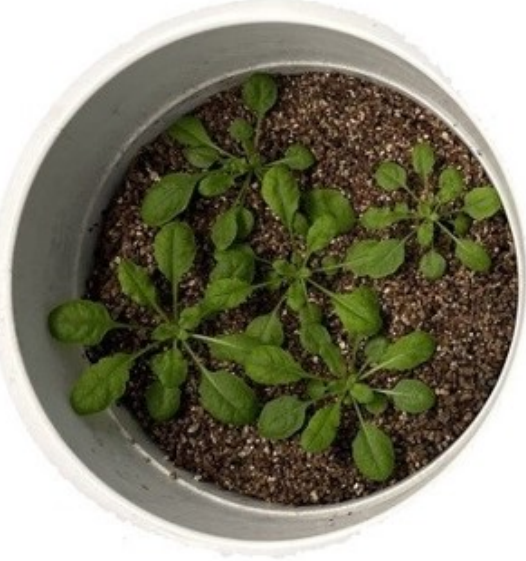

control

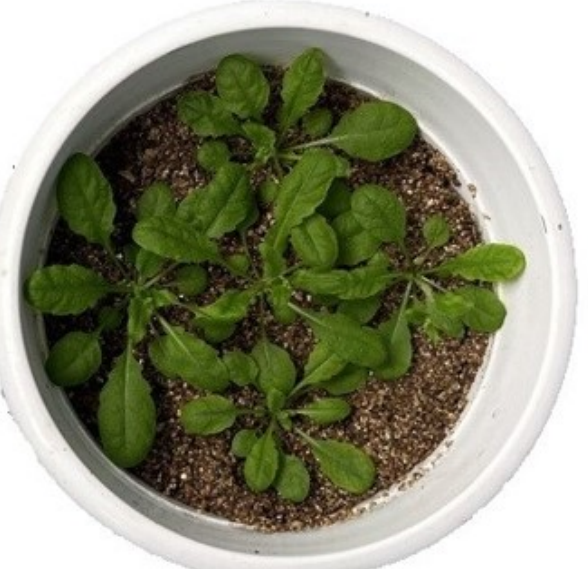

B. aryabhattai

B

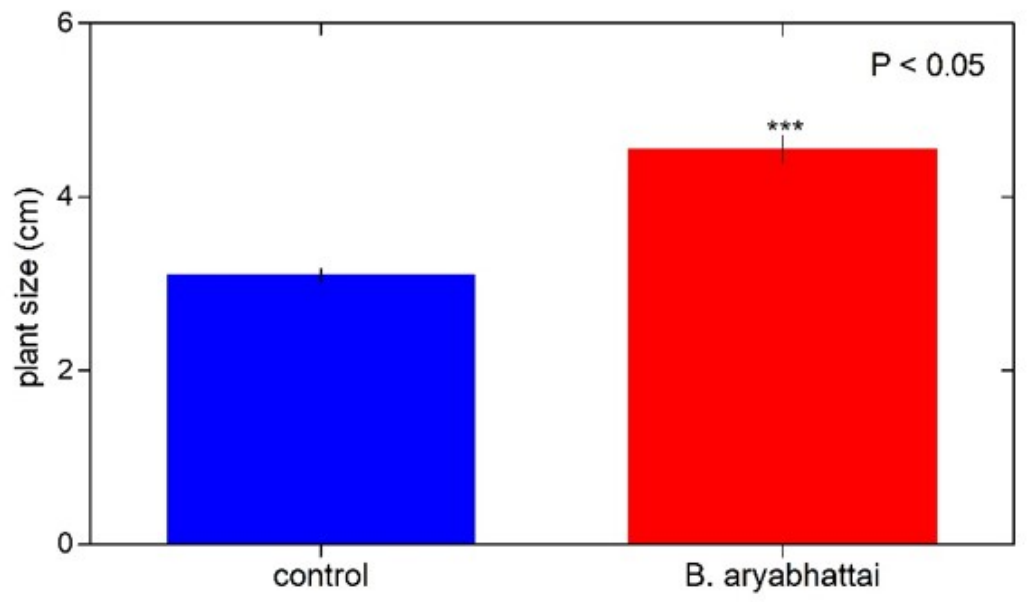

C

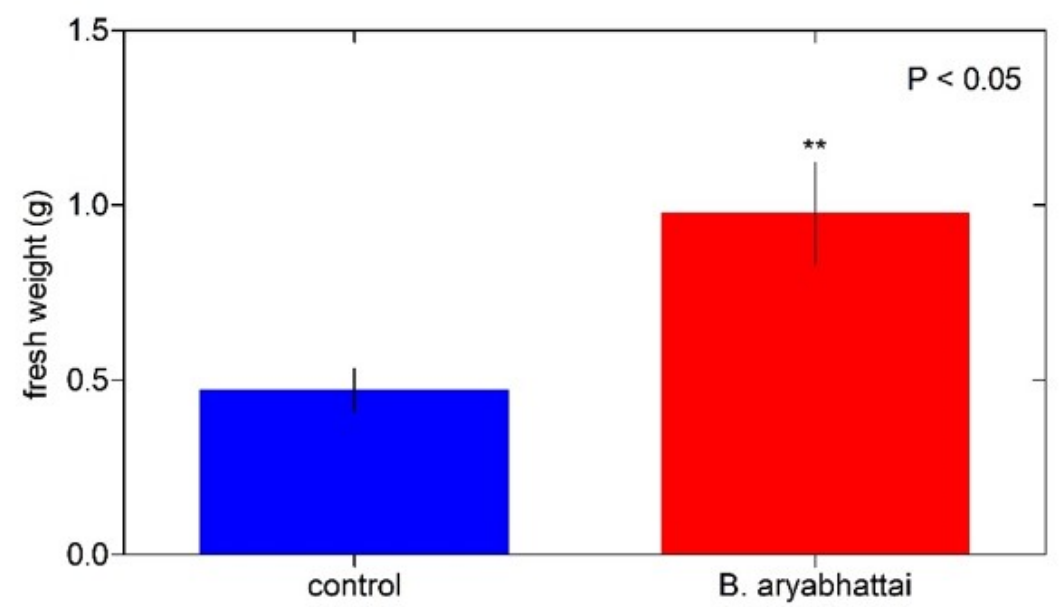

Figure 1

The endophytic bacterium B. aryabhattai enhances Arabidopsis plant growth. A) Phenotype of mocktreated (control) Arabidopsis plants and those treated with B. aryabhattai at 20 days post-inoculation. Plant size (B) and fresh weight (C) of mock-treated Arabidopsis plants and those treated with $B$. aryabhattai. Each bar represents the mean values with standard errors of two independent experiments (n $=15)$ 


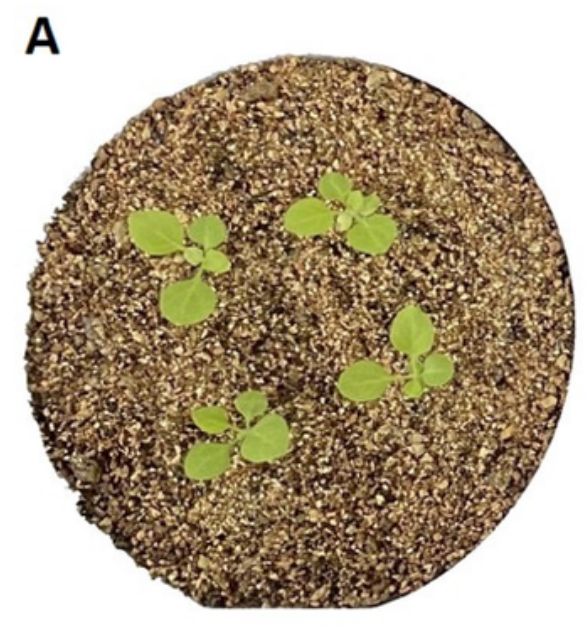

control

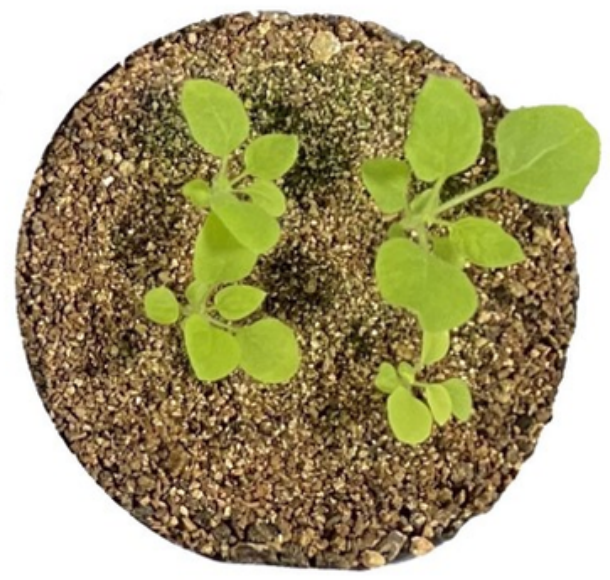

B. aryabhattai

B

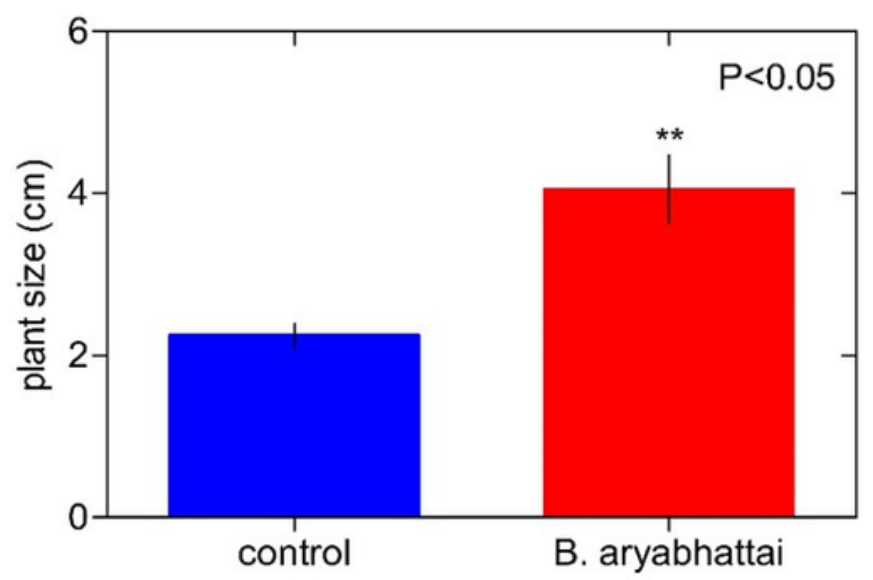

C

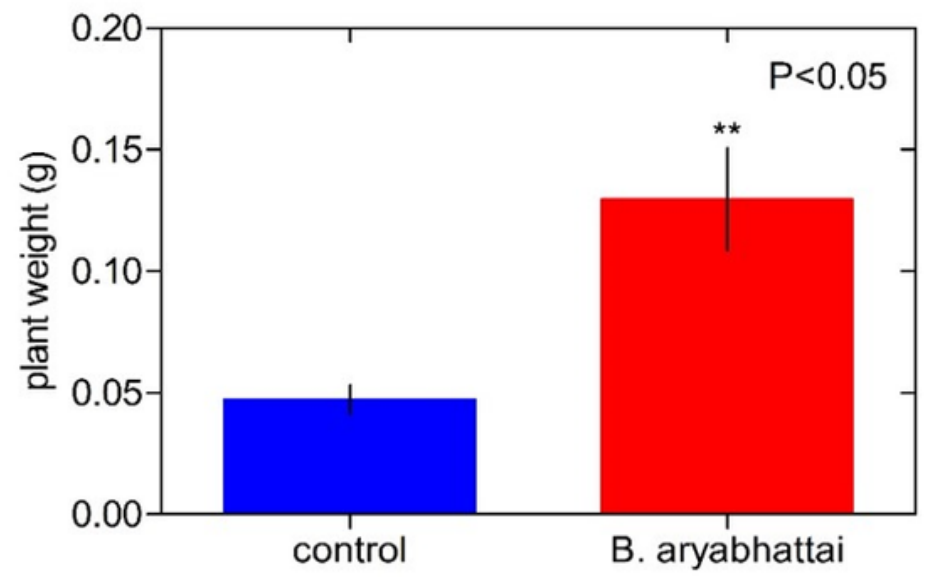

\section{Figure 2}

The endophytic bacterium B. aryabhattai enhances Nicotiana tabacum plant growth. A) Phenotype of mock-treated N. tabacum plants (control) and those treated with B. aryabhattai at 20 days postinoculation. Plant size (B) and fresh weight (C) of mock-treated N. tabacum plants (control) and those treated with $B$. aryabhattai. Each bar represents the mean values with standard errors of two independent experiments $(n=15)$ 


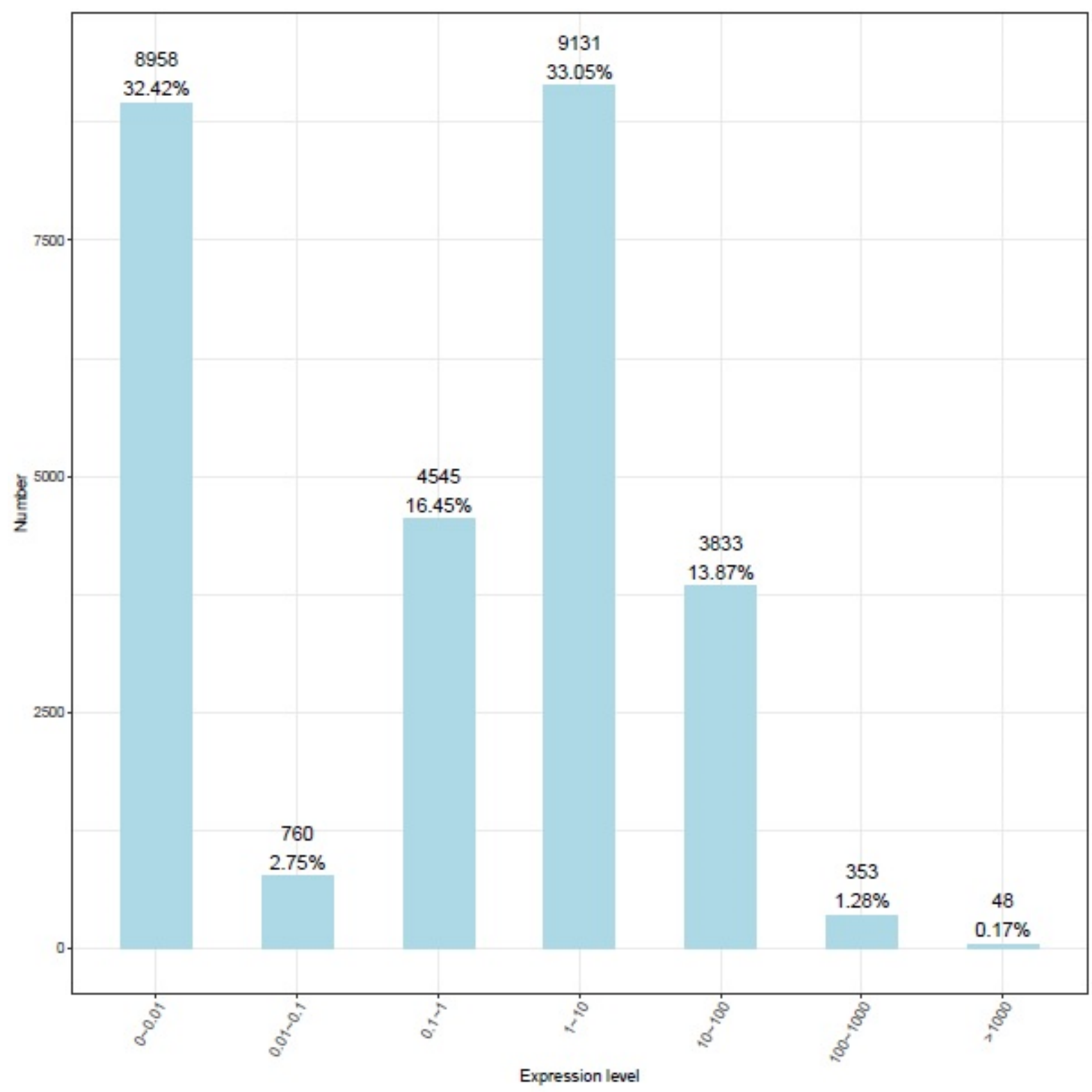

Figure 3

Plant gene expression during the B. aryabhattai - Arabidopsis interaction. The X-axis represents the range of gene expression values, and the $Y$-axis represents the number of genes in each expression interval 


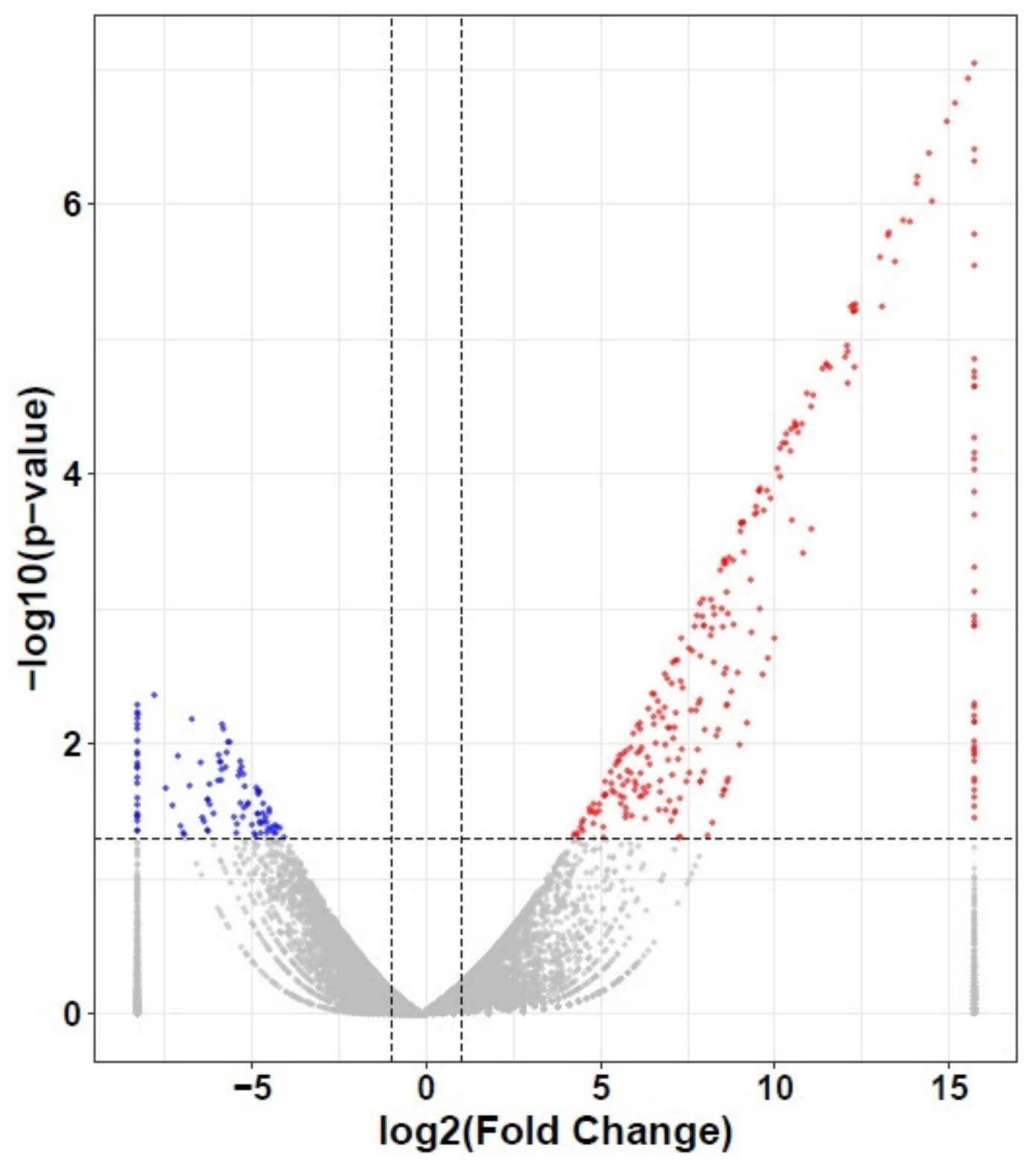

Up (268) Down (95) NoDiff (21053)

\section{Figure 4}

Volcano plot of differential gene expression during the B. aryabhattai - Arabidopsis interaction. The X-axis represents log2 (fold change), and the Y-axis represents - log10 ( $p$ value). The vertical dashed lines represent 2 times the difference threshold, and the horizontal dashed lines are $P$ value thresholds $(0.05)$. The scale was fixed considering the minimum and maximum log2 values (between -7.60 and 15.70 ) 


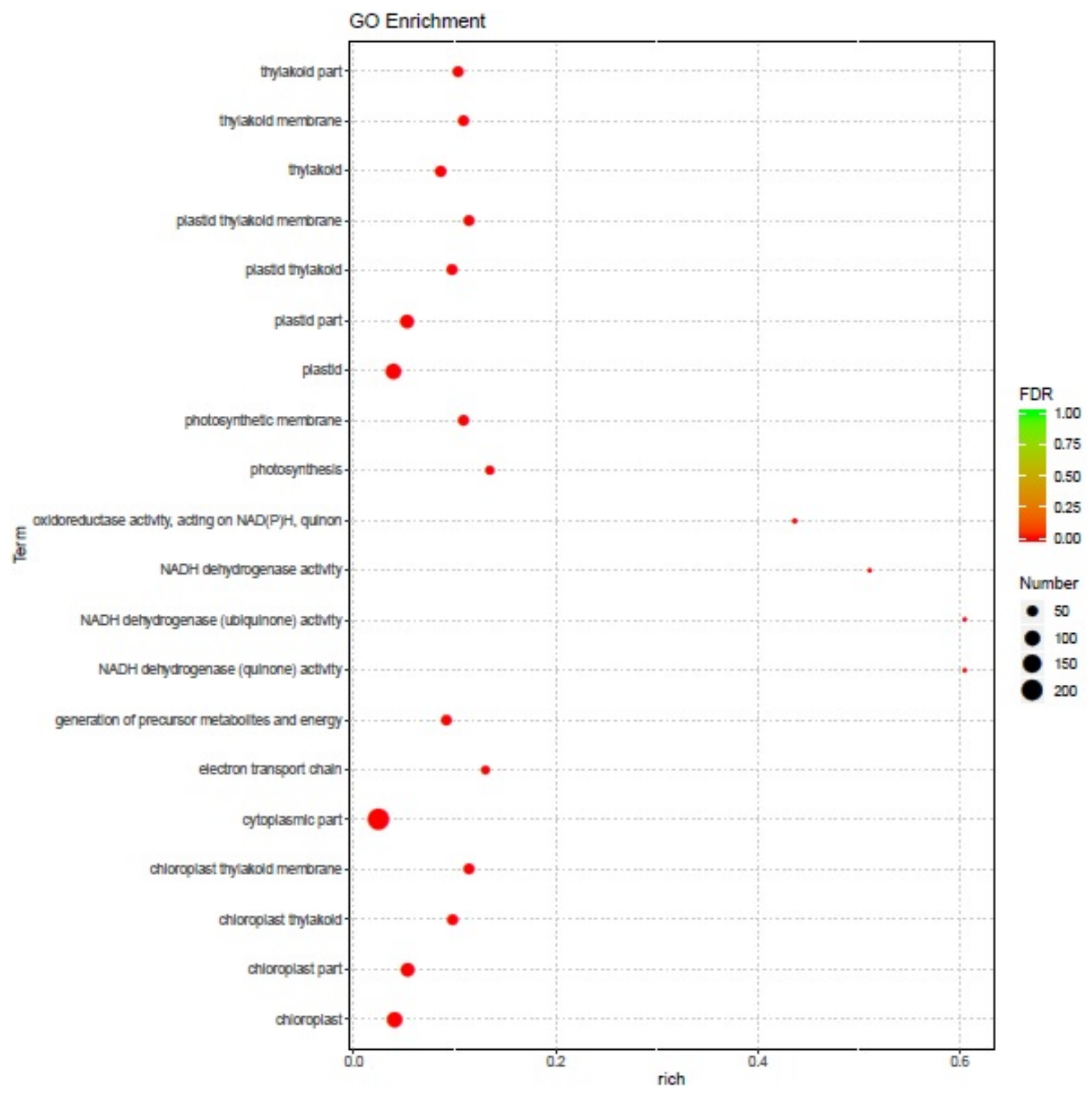

Figure 5

Gene Ontology (GO) enrichment analyses of the differentially expressed genes during the B. aryabhattai Arabidopsis interaction. Based on the GO-rich results, the extent of richness is measured by the rich factor, false discovery rate (FDR) values, and the number of genes that were collected into this GO term. The rich factor refers to the ratio of the number of different genes collected in the GO term to the number of genes annotated. The size of each dot represents the number of enriched genes. The color of each dot is as follows: the red dots indicate pathways with significant enrichment, and the green dots indicate pathways with low enrichment 


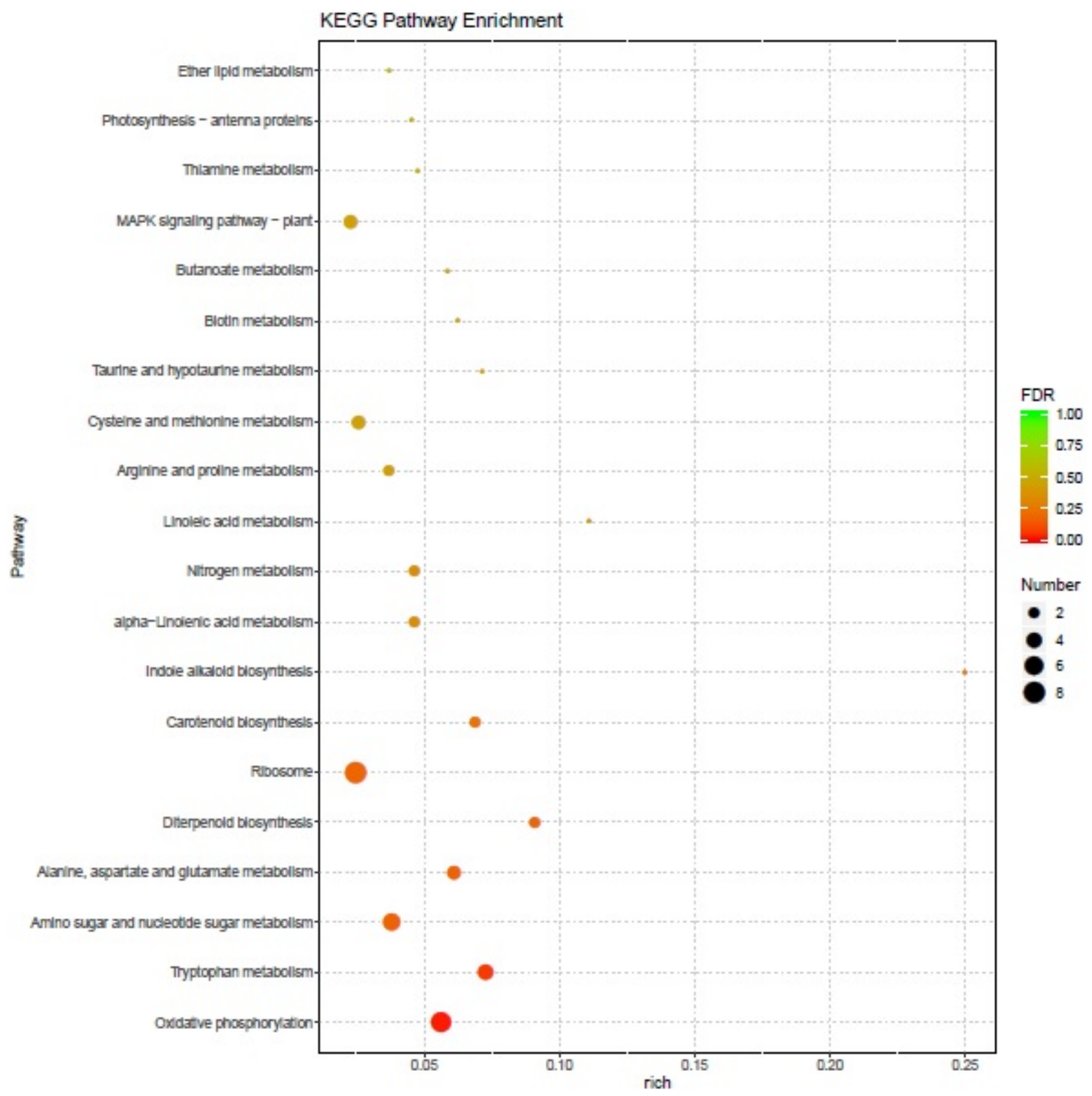

\section{Figure 6}

KEGG pathway analysis of differentially expressed genes during the B. aryabhattai - Arabidopsis interaction. The chart shows the enrichment of differentially expressed genes in signaling pathways. The $\mathrm{Y}$-axis label represents the pathway, and the $\mathrm{X}$-axis label represents the rich factor (rich factor = number of differentially expressed genes enriched in the pathway/number of genes in the background gene set). The size and color of the bubble represent the number of differentially expressed genes enriched in the pathway and enrichment significance, respectively

\section{Supplementary Files}

This is a list of supplementary files associated with this preprint. Click to download.

- TableS1.PlantgeneannotationduringArabidopsisBacillusaryabhattaiinteraction.xlsx 
- TableS2.PlantnewgeneannotationduringArabidopsisBacillusaryabhattaiinteraction.xIsx

- TableS3.Bacillusaryabhattaigeneannotationduringtheinteraction.xlsx 